\title{
A Local Fractional Variational Iteration Method for Laplace Equation within Local Fractional Operators
}

\author{
Yong-Ju Yang, ${ }^{1}$ Dumitru Baleanu, ${ }^{2,3,4}$ and Xiao-Jun Yang ${ }^{5,6}$ \\ ${ }^{1}$ School of Mathematics and Statistics, Nanyang Normal University, Nanyang 4730 61, China \\ ${ }^{2}$ Department of Mathematics and Computer Sciences, Faculty of Arts and Sciences, Cankaya University, 06530 Ankara, Turkey \\ ${ }^{3}$ Department of Chemical and Materials Engineering, Faculty of Engineering, King Abdulaziz University, P.O. Box 80204, \\ Jeddah 21589, Saudi Arabia \\ ${ }^{4}$ Institute of Space Sciences, Magurele, RO-077125 Bucharest, Romania \\ ${ }^{5}$ Institute of Software Science, Zhengzhou Normal University, Zhengzhou 450044, China \\ ${ }^{6}$ Department of Mathematics and Mechanics, China University of Mining and Technology, Xuzhou 221008, China
}

Correspondence should be addressed to Xiao-Jun Yang; dyangxiaojun@163.com

Received 15 November 2012; Accepted 27 February 2013

Academic Editor: Syed Tauseef Mohyud-Din

Copyright (C) 2013 Yong-Ju Yang et al. This is an open access article distributed under the Creative Commons Attribution License, which permits unrestricted use, distribution, and reproduction in any medium, provided the original work is properly cited.

The local fractional variational iteration method for local fractional Laplace equation is investigated in this paper. The operators are described in the sense of local fractional operators. The obtained results reveal that the method is very effective.

\section{Introduction}

As it is known, the partial differential equations $[1,2]$ and fractional differential equations [3-5] appear in many areas of science and engineering. As a result, various kinds of analytical methods and numerical methods were developed [6-8]. For example, the variational iteration method [915] was applied to solve differential equations [16-18], integral equations [19], and numerous applications to different nonlinear equations in physics and engineering. Also, the fractional variational iteration method [20-23] and the fractional complex transform [24-27] were discussed recently. The efficient techniques have successfully addressed a wide class of nonlinear problems for differential equations; see [28-36] and the references therein. We notice that the developed methods are very convenient, efficient, and accurate.

Recently, the local fractional variational iteration method [37] is derived from local fractional operators [38-48]. The method, which accurately computes the solutions in a local fractional series form or in an exact form, presents interest to applied sciences for problems where the other methods cannot be applied properly.

In this paper, we investigate the application of local fractional variational iteration method for solving the local fractional Laplace equations [49] with the different fractal conditions.

This paper is organized as follows.

In Section 2, the basic mathematical tools are reviewed. Section 3 presents briefly the local fractional variational iteration method based on local fractional variational for fractal Lagrange multipliers. Section 4 presents solutions to the local fractional Laplace equations with differential fractal conditions.

\section{Mathematical Fundamentals}

In this section, we present few mathematical fundamentals of local fractional calculus and introduce the basic notions of local fractional continuity, local fractional derivative, and local fractional integral of nondifferential functions. 


\subsection{Local Fractional Continuity}

Lemma 1 (see [42]). Let $F$ be a subset of the real line and $a$ fractal. If $f:(F, d) \rightarrow\left(\Omega^{\prime}, d^{\prime}\right)$ is a bi-Lipschitz mapping, then there is, for constants $\rho, \tau>0$ and $F \subset R$,

$$
\rho^{s} H^{s}(F) \leq H^{s}(f(F)) \leq \tau^{s} H^{s}(F)
$$

such that for all $x_{1}, x_{2} \in F$,

$$
\rho^{\alpha}\left|x_{1}-x_{2}\right|^{\alpha} \leq\left|f\left(x_{1}\right)-f\left(x_{2}\right)\right| \leq \tau^{\alpha}\left|x_{1}-x_{2}\right|^{\alpha} .
$$

As a direct result of Lemma 1, one has [42]

$$
\left|f\left(x_{1}\right)-f\left(x_{2}\right)\right| \leq \tau^{\alpha}\left|x_{1}-x_{2}\right|^{\alpha}
$$

such that

$$
\left|f\left(x_{1}\right)-f\left(x_{2}\right)\right|<\varepsilon^{\alpha},
$$

where $\alpha$ is fractal dimension of $F$.

Suppose that there is [38-43]

$$
\left|f(x)-f\left(x_{0}\right)\right|<\varepsilon^{\alpha}
$$

with $\left|x-x_{0}\right|<\delta$, for $\varepsilon, \delta>0$ and $\varepsilon, \delta \in R$, then $f(x)$ is called local fractional continuous at $x=x_{0}$ and it is denoted by

$$
\lim _{x \rightarrow x_{0}} f(x)=f\left(x_{0}\right) .
$$

Suppose that the function $f(x)$ is satisfied the condition (5) for $x \in(a, b)$, and hence it is called a local fractional continuous on the interval $(a, b)$, denoted by

$$
f(x) \in C_{\alpha}(a, b) .
$$

2.2. Local Fractional Derivatives and Integrals. Suppose that $f(x) \in C_{\alpha}(a, b)$, then the local fractional derivative of $f(x)$ of order $\alpha$ at $x=x_{0}$ is given by [37-43]

$$
\begin{aligned}
D_{x}^{(\alpha)} f\left(x_{0}\right) & =f^{(\alpha)}\left(x_{0}\right)=\left.\frac{d^{\alpha} f(x)}{d x^{\alpha}}\right|_{x=x_{0}} \\
& =\lim _{x \rightarrow x_{0}} \frac{\Delta^{\alpha}\left(f(x)-f\left(x_{0}\right)\right)}{\left(x-x_{0}\right)^{\alpha}},
\end{aligned}
$$

where $\Delta^{\alpha}\left(f(x)-f\left(x_{0}\right)\right) \cong \Gamma(1+\alpha) \Delta\left(f(x)-f\left(x_{0}\right)\right)$.

There is [38-40]

$$
f(x) \in D_{x}^{(\alpha)}(a, b)
$$

if

$$
f^{(\alpha)}(x)=D_{x}^{(\alpha)} f(x)
$$

for any $x \in(a, b)$.

Local fractional derivative of high order is written in the form [38-40]

$$
f^{(k \alpha)}(x)=\overbrace{D_{x}^{(\alpha)} \cdots D_{x}^{(\alpha)}}^{k \text { times }} f(x),
$$

and local fractional partial derivative of high order is [38-40]

$$
\frac{\partial^{k \alpha}}{\partial x^{k \alpha}} f(x)=\overbrace{\frac{\partial^{\alpha}}{\partial x^{\alpha}} \cdots \frac{\partial^{\alpha}}{\partial x^{\alpha}}}^{k \text { times }} f(x) .
$$

Let a function $f(x)$ satisfy the condition (7). Local fractional integral of $f(x)$ of order $\alpha$ in the interval $[a, b]$ is given by [37-43]

$$
\begin{aligned}
{ }_{a} I_{b}^{(\alpha)} f(x) & =\frac{1}{\Gamma(1+\alpha)} \int_{a}^{b} f(t)(d t)^{\alpha} \\
& =\frac{1}{\Gamma(1+\alpha)} \lim _{\Delta t \rightarrow 0} \sum_{j=0}^{j=N-1} f\left(t_{j}\right)\left(\Delta t_{j}\right)^{\alpha},
\end{aligned}
$$

where $\Delta t_{j}=t_{j+1}-t_{j}, \Delta t=\max \left\{\Delta t_{1}, \Delta t_{2}, \Delta t_{j}, \ldots\right\}$, and $\left[t_{j}, t_{j+1}\right], j=0, \ldots, N-1, t_{0}=a, t_{N}=b$, is a partition of the interval $[a, b]$. For other definition of local fractional derivative, see [44-48].

There exists [38-40]

$$
f(x) \in I_{x}^{(\alpha)}(a, b)
$$

if

$$
f^{(\alpha)}(x)={ }_{a} I_{x}^{(\alpha)} f(x)
$$

for any $x \in(a, b)$.

Local fractional multiple integrals of $f(x)$ is written in the form [40]

$$
x_{0} I_{x}^{(k \alpha)} f(x)=\overbrace{x_{0} I_{x}{ }^{(\alpha)} \ldots x_{0} I_{x}{ }^{(\alpha)}}^{k \text { times }} f(x)
$$

if (7) is valid for $x \in(a, b)$.

\section{Local Fractional Variational Iteration Method}

In this section, we introduce the local fractional variational iteration method derived from the local fractional variational approach for fractal Lagrange multipliers [40].

Let us consider the local fractional variational approach in the one-dimensional case through the following local fractional functional, which reads [40]

$$
I(y)={ }_{a} I_{b}{ }^{(\alpha)} f\left(x, y(x), y^{(\alpha)}(x)\right),
$$

where $y^{(\alpha)}(x)$ is taken in local fractional differential operator and $a \leq x \leq b$.

The local fractional variational derivative is given by [40]

$$
\delta^{\alpha} I={ }_{a} I_{b}^{(\alpha)}\left\{\left(\frac{\partial f}{\partial y}-\frac{d^{\alpha}}{d x^{\alpha}}\left(\frac{\partial f}{\partial y^{(\alpha)}}\right)\right) \eta(x)\right\},
$$

where $\delta^{\alpha}$ is local fractional variation signal and $\eta(a)=\eta(b)=$ 0 . 
The nonlinear local fractional equation reads as

$$
L_{\alpha} u+N_{\alpha} u=0
$$

where $L_{\alpha}$ and $N_{\alpha}$ are linear and nonlinear local fractional operators, respectively.

Local fractional variational iteration algorithm can be written as [37]

$$
u_{n+1}(t)=u_{n}(t)+{ }_{t_{0}} I_{t}^{(\alpha)}\left\{\xi^{\alpha}\left[L_{\alpha} u_{n}(s)+N_{\alpha} u_{n}(s)\right]\right\}
$$

Here, we can construct a correction functional as follows [37]:

$$
u_{n+1}(t)=u_{n}(t)+{ }_{t_{0}} I_{t}^{(\alpha)}\left\{\xi^{\alpha}\left[L_{\alpha} u_{n}(s)+N_{\alpha} \widetilde{u}_{n}(s)\right]\right\},
$$

where $\widetilde{u}_{n}$ is considered as a restricted local fractional variation and $\xi^{\alpha}$ is a fractal Lagrange multiplier; that is, $\delta^{\alpha} \widetilde{u}_{n}=0$ $[37,40]$.

Having determined the fractal Lagrangian multipliers, the successive approximations $u_{n+1}, n \geq 0$, of the solution $u$ will be readily obtained upon using any selective fractal function $u_{0}$. Consequently, we have the solution

$$
u=\lim _{n \rightarrow \infty} u_{n}
$$

Here, this technology is called the local fractional variational method [37]. We notice that the classical variation is recovered in case of local fractional variation when the fractal dimension is equal to 1 . Besides, the convergence of local fractional variational process and its algorithms were taken into account [37].

\section{Solutions to Local Fractional Laplace Equation in Fractal Timespace}

The local fractional Laplace equation (see [38-40] and the references therein) is one of the important differential equations with local fractional derivatives. In the following, we consider solutions to local fractional Laplace equations in fractal timespace.

Case 1. Let us start with local fractional Laplace equation given by

$$
\frac{\partial^{2 \alpha} T(x, t)}{\partial t^{2 \alpha}}+\frac{\partial^{2 \alpha} T(x, t)}{\partial x^{2 \alpha}}=0
$$

and subject to the fractal value conditions

$$
\frac{\partial^{\alpha}}{\partial t^{\alpha}} T(x, 0)=0, \quad T(x, 0)=-E_{\alpha}\left(x^{\alpha}\right) .
$$

A corrected local fractional functional for (24) reads as

$$
\begin{aligned}
u_{n+1}(x, t) & \\
= & u_{n}(x, t) \\
& +{ }_{0} I_{t}^{(\alpha)}\left\{\frac{\lambda^{\alpha}}{\Gamma(1+\alpha)}\left(\frac{\partial^{2 \alpha} T_{n}(x, \tau)}{\partial \tau^{2 \alpha}}+\frac{\partial^{2 \alpha} T_{n}(x, \tau)}{\partial x^{2 \alpha}}\right)\right\} .
\end{aligned}
$$

Taking into account the properties of the local fractional derivative, we obtain

$$
\begin{aligned}
& \delta^{\alpha} u_{n+1}(x, t) \\
& =\delta^{\alpha} u_{n}(x, t) \\
& \quad+\delta^{\alpha}{ }_{0} I_{t}{ }^{(\alpha)}\left\{\frac{\lambda^{\alpha}}{\Gamma(1+\alpha)}\left(\frac{\partial^{2 \alpha} T_{n}(x, \tau)}{\partial \tau^{2 \alpha}}+\frac{\partial^{2 \alpha} T_{n}(x, \tau)}{\partial x^{2 \alpha}}\right)\right\} .
\end{aligned}
$$

Hence, from (25)-(26) we get

$$
\begin{aligned}
\delta^{\alpha} u_{n+1}(x, t) & \delta^{\alpha} u_{n}(x, t)+\left.\frac{\lambda^{\alpha}}{\Gamma(1+\alpha)} \delta^{\alpha} u_{n}{ }^{(\alpha)}(x, t)\right|_{\tau=t} \\
& -\left.\left[\frac{\lambda^{\alpha}}{\Gamma(1+\alpha)}\right]^{(\alpha)} \delta^{\alpha} u_{n}(x, t)\right|_{\tau=t} \\
& -\left(\delta^{\alpha} u_{n}(x, \tau)\right){ }_{0} I_{t}^{(\alpha)}\left(\frac{\lambda^{\alpha}}{\Gamma(1+\alpha)}\right)^{(2 \alpha)} \\
= & \delta^{\alpha} u_{n}(x, t)+\left.\frac{\lambda^{\alpha}}{\Gamma(1+\alpha)} \delta^{\alpha} u_{n}{ }^{(\alpha)}\right|_{\tau=t} \\
& -\left.\left(\frac{\lambda^{\alpha}}{\Gamma(1+\alpha)}\right)^{(\alpha)} \delta^{\alpha} u_{n}(x, t)\right|_{\tau=t}{ }^{(2 \alpha)}=0 . \\
& +\left(\delta^{\alpha} u_{n}(x, \tau)\right){ }_{0} I_{t}^{(\alpha)}\left(\frac{\lambda^{\alpha}}{\Gamma(1+\alpha)}\right)^{(1+\alpha)}
\end{aligned}
$$

As a result, from (27) we can derive

$$
\begin{gathered}
\left(\frac{\lambda^{\alpha}}{\Gamma(1+\alpha)}\right)^{(2 \alpha)}=0,\left.\quad \frac{\lambda^{\alpha}}{\Gamma(1+\alpha)}\right|_{\tau=t}=0 \\
\left(\frac{\lambda^{\alpha}}{\Gamma(1+\alpha)}\right)^{(\alpha)}=1 .
\end{gathered}
$$

We have $\lambda=\tau-t$ such that the fractal Lagrange multiplier reads as

$$
\frac{\lambda^{\alpha}}{\Gamma(1+\alpha)}=\frac{(\tau-t)^{\alpha}}{\Gamma(1+\alpha)}
$$

From (24) we take the initial value, which reads as

$$
u_{0}(x, t)=-E_{\alpha}\left(x^{\alpha}\right) .
$$

By using (25) we structure a local fractional iteration procedure as

$$
\begin{aligned}
u_{n+1}(x, t) & \\
= & u_{n}(x, t) \\
& +{ }_{0} I_{t}{ }^{(\alpha)}\left\{\frac{(\tau-t)^{\alpha}}{\Gamma(1+\alpha)}\left(\frac{\partial^{2 \alpha} T_{n}(x, \tau)}{\partial \tau^{2 \alpha}}+\frac{\partial^{2 \alpha} T_{n}(x, \tau)}{\partial x^{2 \alpha}}\right)\right\} .
\end{aligned}
$$


Hence, we can derive the first approximation term as

$$
\begin{aligned}
u_{1}(x, t) & \\
= & u_{0}(x, t) \\
& +{ }_{0} I_{t}^{(\alpha)}\left\{\frac{(\tau-t)^{\alpha}}{\Gamma(1+\alpha)}\left(\frac{\partial^{2 \alpha} T_{0}(x, \tau)}{\partial \tau^{2 \alpha}}+\frac{\partial^{2 \alpha} T_{0}(x, \tau)}{\partial x^{2 \alpha}}\right)\right\} \\
= & -E_{\alpha}\left(x^{\alpha}\right)+{ }_{0} I_{t}^{(\alpha)}\left\{\frac{(\tau-t)^{\alpha}}{\Gamma(1+\alpha)}\left(-E_{\alpha}\left(x^{\alpha}\right)\right)\right\} \\
= & E_{\alpha}\left(x^{\alpha}\right)\left(-1+\frac{t^{2 \alpha}}{\Gamma(1+2 \alpha)}\right) .
\end{aligned}
$$

The second approximation can be calculated in the similar way, which is

$$
\begin{aligned}
u_{2}(x, t) & \\
= & u_{1}(x, t) \\
& +{ }_{0} I_{t}{ }^{(\alpha)}\left\{\frac{(\tau-t)^{\alpha}}{\Gamma(1+\alpha)}\left(\frac{\partial^{2 \alpha} T_{1}(x, \tau)}{\partial \tau^{2 \alpha}}+\frac{\partial^{2 \alpha} T_{1}(x, \tau)}{\partial x^{2 \alpha}}\right)\right\} \\
= & E_{\alpha}\left(x^{\alpha}\right)\left(-1+\frac{t^{2 \alpha}}{\Gamma(1+2 \alpha)}\right) \\
& +{ }_{0} I_{t}{ }^{(\alpha)}\left\{\frac{(\tau-t)^{\alpha}}{\Gamma(1+\alpha)}\left(\frac{t^{2 \alpha} E_{\alpha}\left(x^{\alpha}\right)}{\Gamma(1+2 \alpha)}\right)\right\} \\
= & E_{\alpha}\left(x^{\alpha}\right)\left(-1+\frac{t^{2 \alpha}}{\Gamma(1+2 \alpha)}-\frac{t^{4 \alpha}}{\Gamma(1+4 \alpha)}\right) .
\end{aligned}
$$

Proceeding in this manner, we get

$$
u_{n}(x, t)=E_{\alpha}\left(x^{\alpha}\right)\left(\sum_{k=0}^{n}(-1)^{k} \frac{t^{2 k \alpha}}{\Gamma(1+2 k \alpha)}\right) .
$$

Thus, the final solution reads as

$$
\begin{aligned}
u(x, t) & =\lim _{n \rightarrow \infty} u_{n}(x, t) \\
& =E_{\alpha}\left(x^{\alpha}\right)\left(\sum_{k=0}^{\infty}(-1)^{k} \frac{t^{2 k \alpha}}{\Gamma(1+2 k \alpha)}\right) \\
& =-E_{\alpha}\left(x^{\alpha}\right) \cos _{\alpha}\left(t^{\alpha}\right) .
\end{aligned}
$$

Case 2. Consider the local fractional Laplace equation as

$$
\frac{\partial^{2 \alpha} T(x, t)}{\partial t^{2 \alpha}}+\frac{\partial^{2 \alpha} T(x, t)}{\partial x^{2 \alpha}}=0
$$

subject to fractal value conditions given by

$$
\frac{\partial^{\alpha}}{\partial t^{\alpha}} T(x, 0)=-E_{\alpha}\left(x^{\alpha}\right), \quad T(x, 0)=0 .
$$

Now we can structure the same local fractional iteration procedure (31).
By using (36)-(37) we take an initial value as

$$
u_{0}(x, t)=-\frac{t^{\alpha} E_{\alpha}\left(x^{\alpha}\right)}{\Gamma(1+\alpha)} .
$$

The first approximation term reads as

$$
\begin{aligned}
u_{1}(x, t) & \\
= & u_{0}(x, t) \\
& +{ }_{0} I_{t}{ }^{(\alpha)}\left\{\frac{(\tau-t)^{\alpha}}{\Gamma(1+\alpha)}\left(\frac{\partial^{2 \alpha} T_{0}(x, \tau)}{\partial \tau^{2 \alpha}}+\frac{\partial^{2 \alpha} T_{0}(x, \tau)}{\partial x^{2 \alpha}}\right)\right\} \\
= & -\frac{t^{\alpha} E_{\alpha}\left(x^{\alpha}\right)}{\Gamma(1+\alpha)}+{ }_{0} I_{t}^{(\alpha)}\left\{\frac{(\tau-t)^{\alpha}}{\Gamma(1+\alpha)}\left(-\frac{t^{\alpha} E_{\alpha}\left(x^{\alpha}\right)}{\Gamma(1+\alpha)}\right)\right\} \\
= & -\frac{t^{\alpha} E_{\alpha}\left(x^{\alpha}\right)}{\Gamma(1+\alpha)}+\frac{t^{3 \alpha} E_{\alpha}\left(x^{\alpha}\right)}{\Gamma(1+3 \alpha)} .
\end{aligned}
$$

In the same manner, the second approximation is given by

$$
\begin{aligned}
u_{2}(x, t) & \\
= & u_{1}(x, t) \\
& +{ }_{0} I_{t}{ }^{(\alpha)}\left\{\frac{(\tau-t)^{\alpha}}{\Gamma(1+\alpha)}\left(\frac{\partial^{2 \alpha} T_{1}(x, \tau)}{\partial \tau^{2 \alpha}}+\frac{\partial^{2 \alpha} T_{1}(x, \tau)}{\partial x^{2 \alpha}}\right)\right\} \\
= & -\frac{t^{\alpha} E_{\alpha}\left(x^{\alpha}\right)}{\Gamma(1+\alpha)}+\frac{t^{3 \alpha} E_{\alpha}\left(x^{\alpha}\right)}{\Gamma(1+3 \alpha)} \\
& +{ }_{0} I_{t}{ }^{(\alpha)}\left\{\frac{(\tau-t)^{\alpha}}{\Gamma(1+\alpha)}\left(\frac{t^{3 \alpha} E_{\alpha}\left(x^{\alpha}\right)}{\Gamma(1+3 \alpha)}\right)\right\} \\
= & -\frac{t^{\alpha} E_{\alpha}\left(x^{\alpha}\right)}{\Gamma(1+\alpha)}+\frac{t^{3 \alpha} E_{\alpha}\left(x^{\alpha}\right)}{\Gamma(1+3 \alpha)}-\frac{t^{5 \alpha} E_{\alpha}\left(x^{\alpha}\right)}{\Gamma(1+5 \alpha)} .
\end{aligned}
$$

Finally, we can obtain the local fractional series solution as follows:

$$
u_{n}(x, t)=E_{\alpha}\left(x^{\alpha}\right)\left(\sum_{k=0}^{n}(-1)^{k} \frac{t^{(2 k+1) \alpha}}{\Gamma(1+(2 k+1) \alpha)}\right) .
$$

Thus, the expression of the final solution is given by

$$
\begin{aligned}
u(x, t) & =\lim _{n \rightarrow \infty} u_{n}(x, t) \\
& =E_{\alpha}\left(x^{\alpha}\right)\left(\sum_{i=0}^{\infty}(-1)^{k} \frac{t^{(2 k+1) \alpha}}{\Gamma(1+(2 k+1) \alpha)}\right) \\
& =-E_{\alpha}\left(x^{\alpha}\right) \sin _{\alpha}\left(t^{\alpha}\right) .
\end{aligned}
$$

As is known, the Mittag-Leffler function in fractal space can be written in the form

$$
\begin{gathered}
\left|E_{\alpha}\left(x^{\alpha}\right)-E_{\alpha}\left(x_{0}^{\alpha}\right)\right| \leq E_{\alpha}\left(x_{0}^{\alpha}\right)\left|x-x_{0}\right|^{\alpha}<\varepsilon^{\alpha}, \\
\left|\sin _{\alpha}\left(t^{\alpha}\right)-\sin _{\alpha}\left(t_{0}^{\alpha}\right)\right|<\left|\cos _{\alpha}\left(x_{0}^{\alpha}\right)\right|\left|t-t_{0}\right|^{\alpha}<\varepsilon^{\alpha} .
\end{gathered}
$$

Hence, the fractal dimensions of both $E_{\alpha}\left(x^{\alpha}\right)$ and $\cos _{\alpha}\left(t^{\alpha}\right)$ are equal to $\alpha$. 


\section{Conclusions}

Local fractional calculus is set up on fractals and the local fractional variational iteration method is derived from local fractional calculus. This new technique is efficient for the applied scientists to process these differential and integral equations with the local fractional operators. The variational iteration method $[9-19,27]$ is derived from fractional calculus and classical calculus; the fractional variational iteration method $[20-22,27]$ is derived from the modified fractional derivative, while the local fractional variational iteration method [37] is derived from the local fractional calculus [3743]. Other methods for local fractional ordinary and partial differential equations were considered in [27].

In this paper, two outstanding examples of applications of the local fractional variational iteration method to the local fractional Laplace equations are investigated in detail. The reliable obtained results are complementary with the ones presented in the literature.

\section{References}

[1] R. D. Driver, Ordinary and Delay Differential Equations, Springer, Berlin, Germany, 1977.

[2] A. M. Wazwaz, Partial Differential Equations: Methods and Applications, Balkema Publishers, Leiden, The Netherlands, 2002.

[3] I. Podlubny, Fractional Differential Equations, Academic Press, New York, NY, USA, 1999.

[4] R. Hilfer, Applications of Fractional Calculus in Physics, World Scientific Publishing, Singapore, 2000.

[5] A. A. Kilbas, H. M. Srivastava, and J. J. Trujillo, Theory and Applications of Fractional Differential Equations, Elsevier, Amsterdam, The Netherlands, 2006.

[6] G. Adomian, Solving Frontier Problems of Physics: The Decomposition Method, Kluwer Academic Publishers, Boston, Mass, USA, 1994.

[7] D. Baleanu, K. Diethelm, E. Scalas, and J. J. Trujillo, Fractional Calculus Models and Numerical Methods, vol. 3 of Series on Complexity, Nonlinearity and Chaos, World Scientific Publishing, 2012.

[8] A.-M. Wazwaz, A First Course in Integral Equations, World Scientific Publishing, Singapore, 1997.

[9] J.-H. He, "Variational iteration method for autonomous ordinary differential systems," Applied Mathematics and Computation, vol. 114, no. 2-3, pp. 115-123, 2000.

[10] J.-H. He, "Variational approach for nonlinear oscillators," Chaos, Solitons \& Fractals, vol. 34, no. 5, pp. 1430-1439, 2007.

[11] J. H. He, "Variational iteration method-a kind of non-linear analytical technique: some examples," International Journal of Non-Linear Mechanics, vol. 34, no. 4, pp. 699-708, 1999.

[12] J. H. He, "Comment on variational iteration method for fractional calculus using He's polynomials," Abstract and Applied Analysis, vol. 2012, Article ID 964974, 2 pages, 2012.

[13] S. T. Mohyud-Din, M. A. Noor, K. I. Noor, and M. M. Hosseini, "Solution of singular equation by He's variational iteration method," International Journal of Nonlinear Sciences and Numerical Simulation, vol. 11, no. 2, pp. 81-86, 2010.

[14] J.-H. He, "Some asymptotic methods for strongly nonlinear equations," International Journal of Modern Physics B, vol. 20, no. 10, pp. 1141-1199, 2006.
[15] J. H. He, G. C. Wu, and F. Austin, "The variational iteration method which should be followed," Nonlinear Science Letters A, vol. 1, no. 1, pp. 1-30, 2010.

[16] M. Tatari and M. Dehghan, "He's variational iteration method for computing a control parameter in a semi-linear inverse parabolic equation," Chaos, Solitons \& Fractals, vol. 33, no. 2, pp. 671-677, 2007.

[17] S. Momani and S. Abuasad, "Application of He's variational iteration method to Helmholtz equation," Chaos, Solitons \& Fractals, vol. 27, no. 5, pp. 1119-1123, 2006.

[18] Z. M. Odibat and S. Momani, "Application of variational iteration method to nonlinear differential equations of fractional order," International Journal of Nonlinear Sciences and Numerical Simulation, vol. 7, no. 1, pp. 27-34, 2006.

[19] L. Xu, "Variational iteration method for solving integral equations," Computers \& Mathematics with Applications, vol. 54, no. 7-8, pp. 1071-1078, 2007.

[20] J.-H. He, "A short remark on fractional variational iteration method," Physics Letters A, vol. 375, no. 38, pp. 3362-3364, 2011.

[21] G.-c. Wu and E. W. M. Lee, "Fractional variational iteration method and its application," Physics Letters A, vol. 374, no. 25, pp. 2506-2509, 2010.

[22] Y. Khan, N. Faraz, A. Yildirim, and Q. Wu, "Fractional variational iteration method for fractional initial-boundary value problems arising in the application of nonlinear science," Computers \& Mathematics with Applications, vol. 62, no. 5, pp. 2273-2278, 2011.

[23] J.-H. He, S. K. Elagan, and Z. B. Li, "Geometrical explanation of the fractional complex transform and derivative chain rule for fractional calculus," Physics Letters A, vol. 376, no. 4, pp. 257259,2012

[24] Z. B. Li and J. H. He, "Application of the fractional complex transform to fractional differential equations," Nonlinear Science Letters A, vol. 2, no. 3, pp. 121-126, 2011.

[25] Z. B. Li, W. H. Zhu, and J. H. He, "Exact solutions of timefractional heat conduction equation by the fractional complex transform," Thermal Science, vol. 16, no. 2, pp. 335-338, 2012.

[26] Q. L. Wang, J. H. He, and Z. B. Li, "Fractional model for heat conduction in polar bear hairs," Thermal Science, vol. 16, no. 2, pp. 339-342, 2012.

[27] J. H. He, "Asymptotic methods for solitary solutions and compactons," Abstract and Applied Analysis, vol. 2012, Article ID 916793, 130 pages, 2012.

[28] S. T. Mohyud-Din, A. Yildirim, and G. Demirli, "Analytical solution of wave system in $R^{n}$ with coupling controllers," International Journal of Numerical Methods for Heat \& Fluid Flow, vol. 21, no. 2, pp. 198-205, 2011.

[29] S. T. Mohyud-Din, A. Yıldırım, and S. Sarıaydın, "Numerical soliton solution of the Kaup-Kupershmidt equation," International Journal of Numerical Methods for Heat \& Fluid Flow, vol. 21, no. 3-4, pp. 272-281, 2011.

[30] S. T. Mohyud-Din, A. Yıldırım, and S. A. Sezer, "Numerical soliton solutions of improved Boussinesq equation," International Journal of Numerical Methods for Heat \& Fluid Flow, vol. 21, no. 6-7, pp. 822-827, 2011.

[31] M. A. Abdou, A. A. Soliman, and S. T. El-Basyony, "New application of Exp-function method for improved Boussinesq equation," Physics Letters A, vol. 369, no. 5-6, pp. 469-475, 2007.

[32] S. A. El-Wakil, M. A. Madkour, and M. A. Abdou, "Application of Exp-function method for nonlinear evolution equations with variable coefficients," Physics Letters A, vol. 369, no. 1-2, pp. 6269, 2007. 
[33] J. H. He, "An elementary introduction to recently developed asymptotic methods and nanomechanics in textile engineering," International Journal of Modern Physics B, vol. 22, no. 21, pp. 3487-3578, 2008.

[34] S. T. Mohyud-Din, M. A. Noor, and K. I. Noor, "Some relatively new techniques for nonlinear problems," Mathematical Problems in Engineering, vol. 2009, Article ID 234849, 25 pages, 2009.

[35] W.-X. Ma, H. Wu, and J. He, "Partial differential equations possessing Frobenius integrable decompositions," Physics Letters A, vol. 364, no. 1, pp. 29-32, 2007.

[36] W. X. Ma and Y. You, "Rational solutions of the Toda lattice equation in Casoratian form," Chaos, Solitons and Fractals, vol. 22, no. 2, pp. 395-406, 2004.

[37] X. J. Yang and D. Baleanu, "Fractal heat conduction problem solved by local fractional variation iteration method," Thermal Science, 2012.

[38] X. J. Yang, "Local fractional integral transforms," Progress in Nonlinear Science, vol. 4, pp. 1-225, 2011.

[39] X. J. Yang, Local Fractional Functional Analysis and Its Applications, Asian Academic Publisher, Hong Kong, 2011.

[40] X. J. Yang, Advanced Local Fractional Calculus and Its Applications, World Science Publisher, New York, NY, USA, 2012.

[41] W. P. Zhong, X. J. Yang, and F. Gao, "A Cauchy problem for some local fractional abstract differential equation with fractal conditions," Journal of Applied Functional Analysis, vol. 8, no. 1, pp. 92-99, 2013.

[42] M. S. Hu, R. P. Agarwal, and X. J. Yang, "Local fractional Fourier series with application to wave equation in fractal vibrating string," Abstract and Applied Analysis, vol. 2012, Article ID 567401, 15 pages, 2012.

[43] M. S. Hu, D. Baleanu, and X. J. Yang, "One-phase problems for discontinuous heat transfer in fractal media," Mathematical Problems in Engineering, vol. 2013, Article ID 358473, 3 pages, 2013.

[44] K. M. Kolwankar and A. D. Gangal, "Fractional differentiability of nowhere differentiable functions and dimensions," Chaos, vol. 6, no. 4, pp. 505-513, 1996.

[45] J. H. He, "A new fractal derivation," Thermal Science, vol. 15, pp. S145-S147, 2011.

[46] W. Chen, "Time-space fabric underlying anomalous diffusion," Chaos, Solitons \& Fractals, vol. 28, no. 4, pp. 923-929, 2006.

[47] J. Fan and J. H. He, "Biomimic design of multi-scale fabric with efficient heat transfer property," Thermal Science, vol. 16, no. 5, pp. 1349-1352, 2012.

[48] J. Fan and J. H. He, "Fractal derivative model for air permeability in hierarchic porous media," Abstract and Applied Analysis, vol. 2012, Article ID 354701, 7 pages, 2012.

[49] A. Liangprom and K. Nonlaopon, "On the convolution equation related to the diamond Klein-Gordon operator," Abstract and Applied Analysis, vol. 2011, Article ID 908491, 16 pages, 2011. 


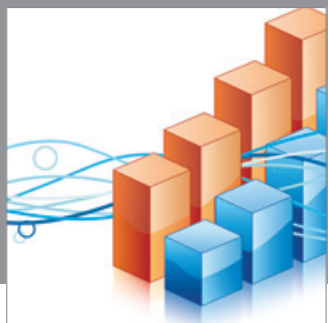

Advances in

Operations Research

mansans

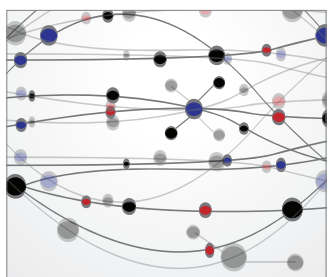

The Scientific World Journal
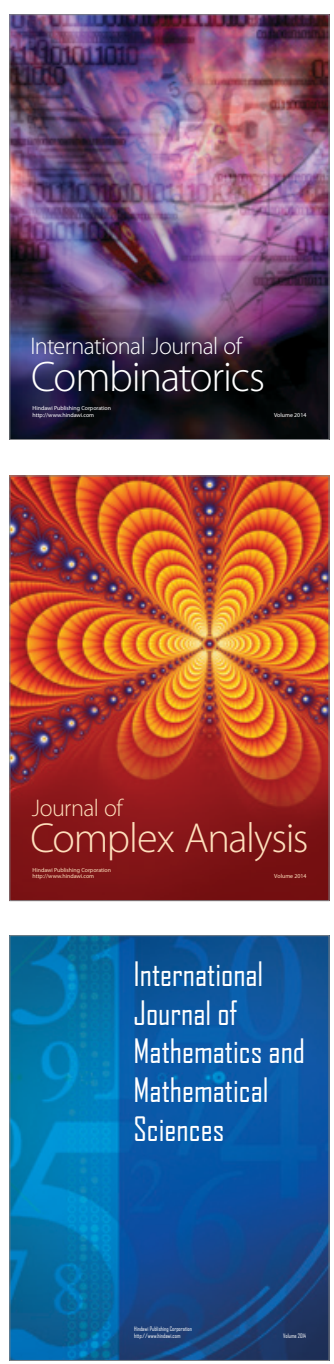
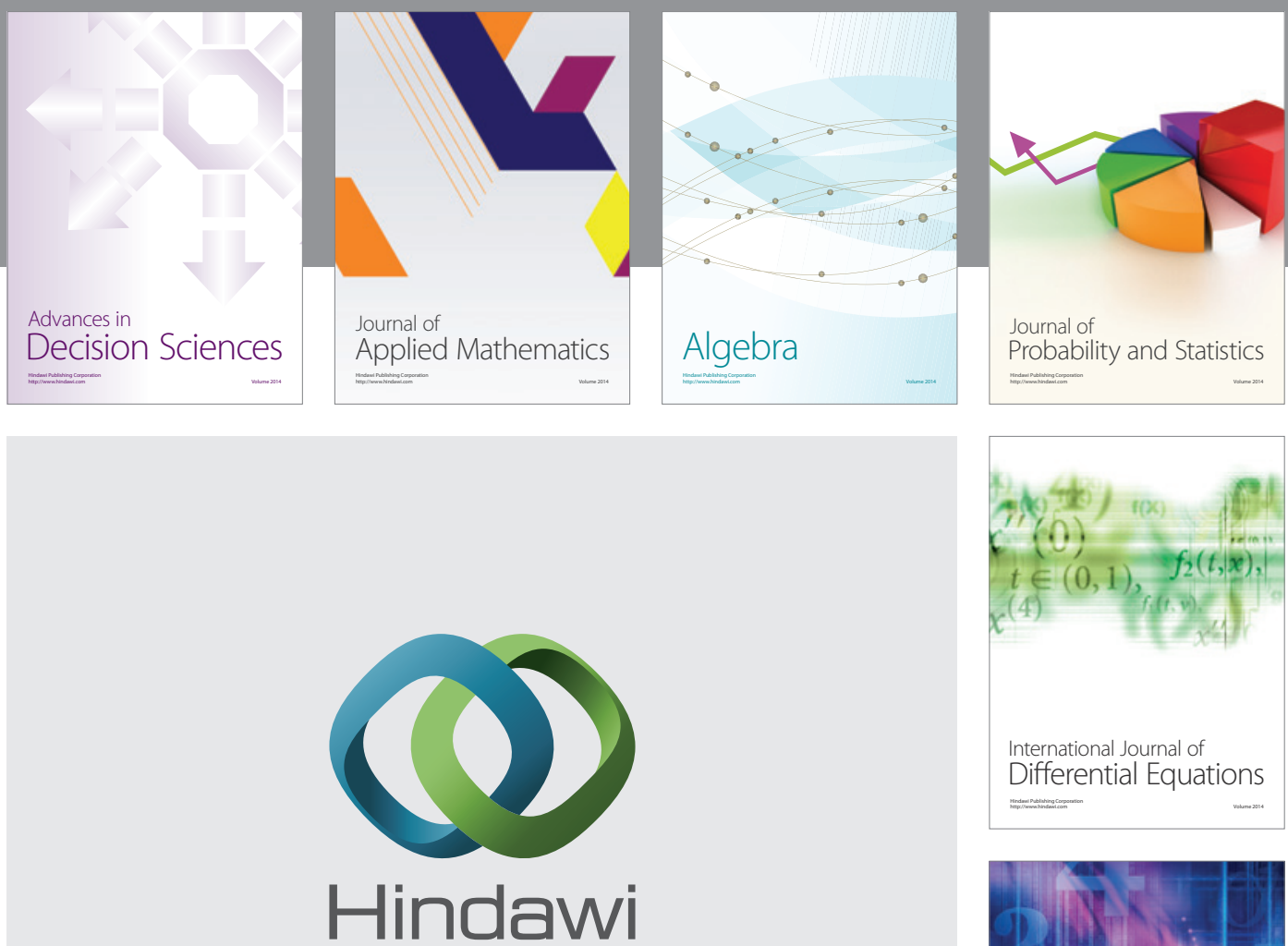

Submit your manuscripts at http://www.hindawi.com
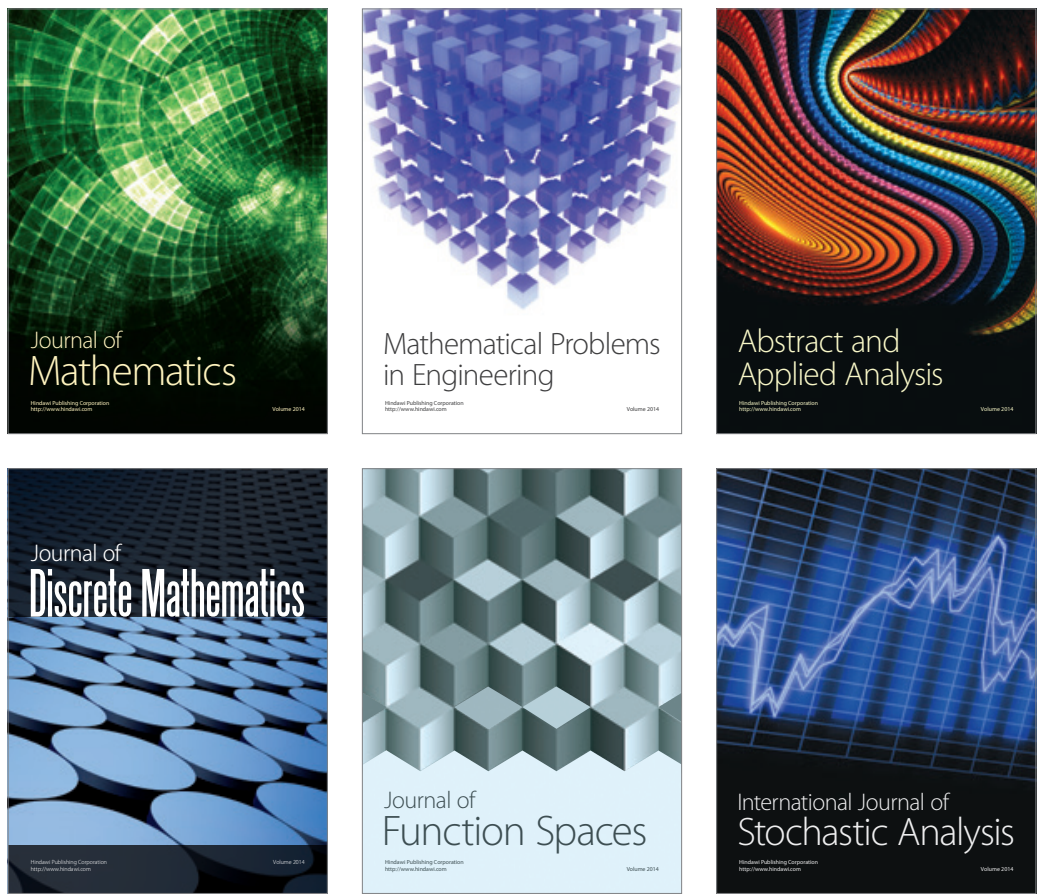

Journal of

Function Spaces

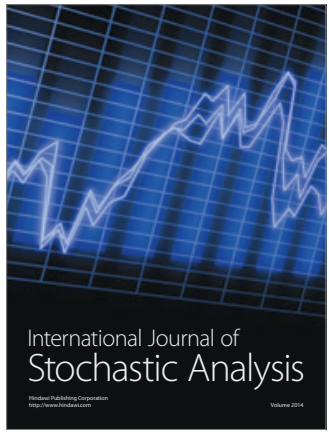

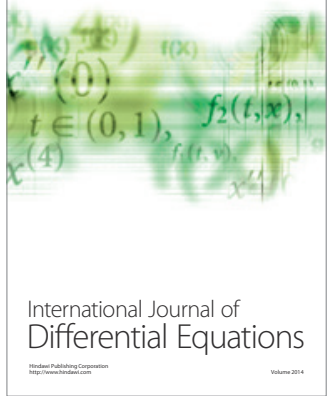
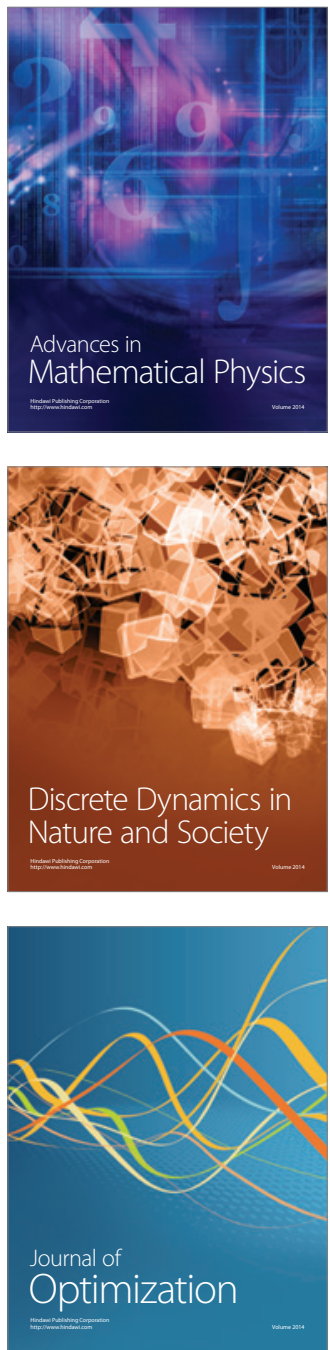